\title{
Novel Formulations of Antiviral Drug Favipiravir: Improving Permeability and Tabletability
}

Xinyuan Wang, Lei Wang,* Changlin Yao, Guanying Xie, Shuhong Song, Huimin Li, Yaqian Qu, and Xutang Tao*

State Key Laboratory of Crystal Materials, Shandong University, Jinan, 250100, China

E-mail: icmwl@sdu.edu.cn; txt@sdu.edu.cn 


\section{Surporting information}

\section{Supplementary Figures}

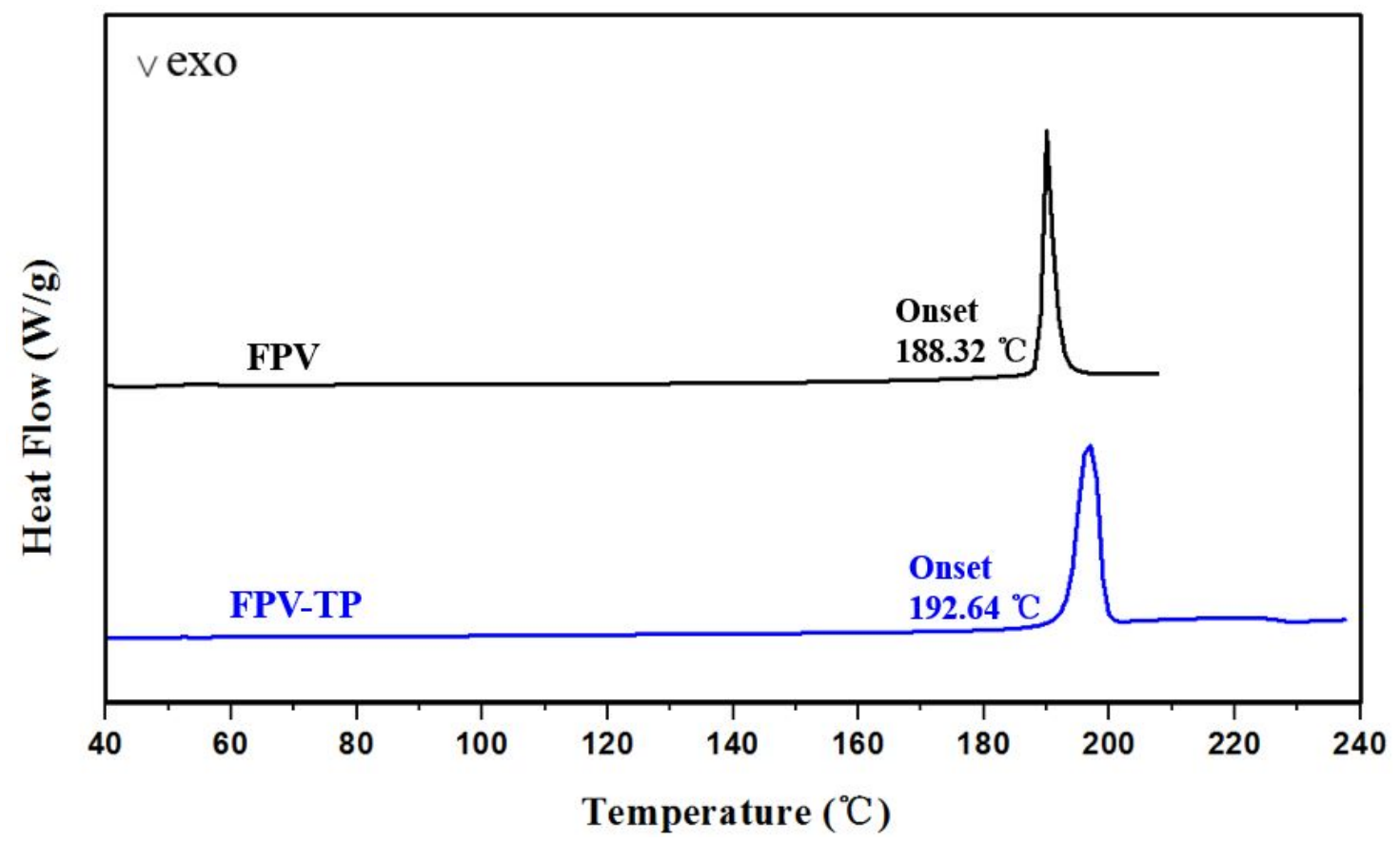

Figure S1. DSC thermograms of FPV and FPV-TP. 

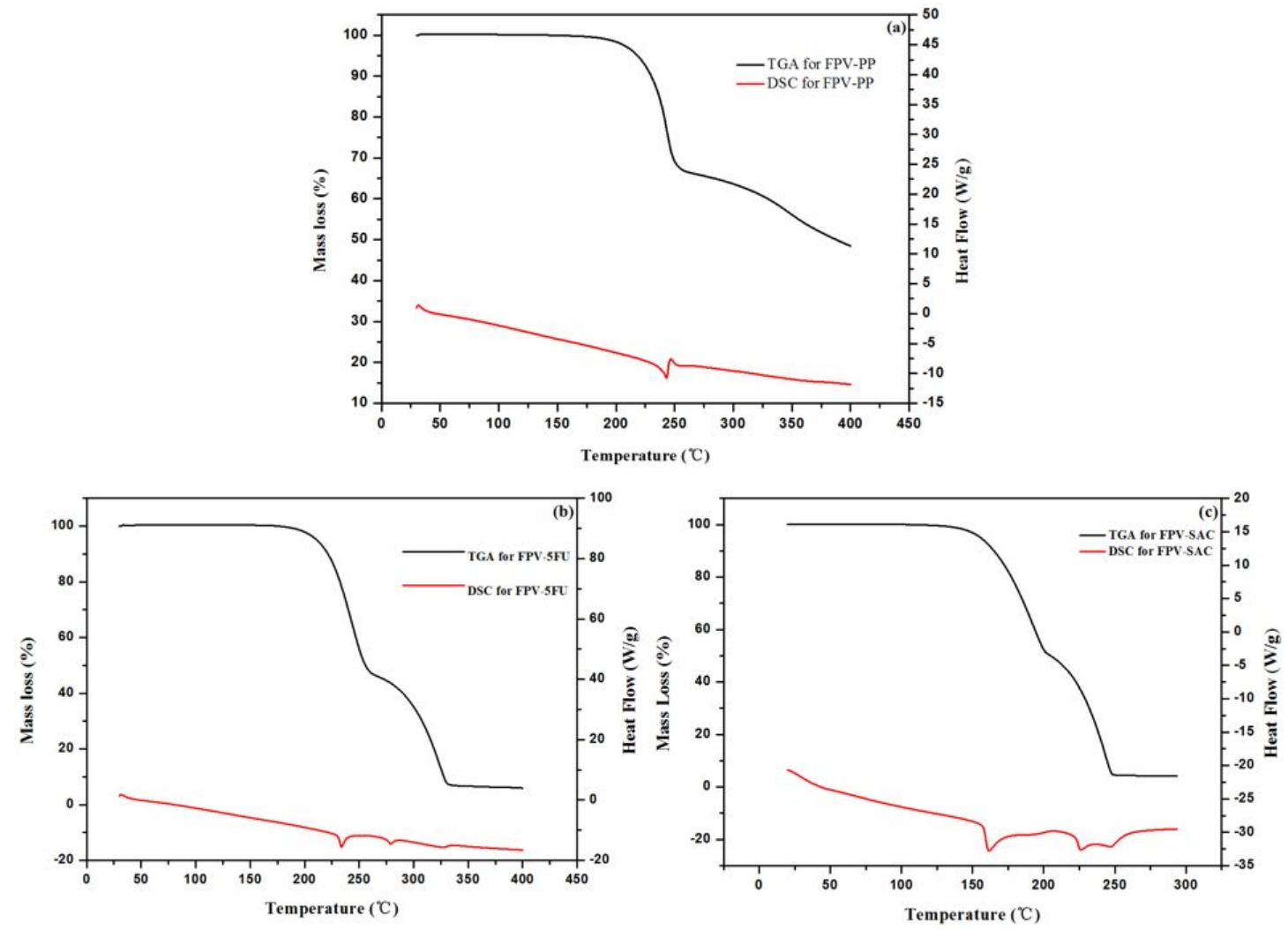

Figure S2. Superposed TGA (red lines) and DSC (blue lines) analyses of FPV-PP, FPV5FU and FPV-SAC.

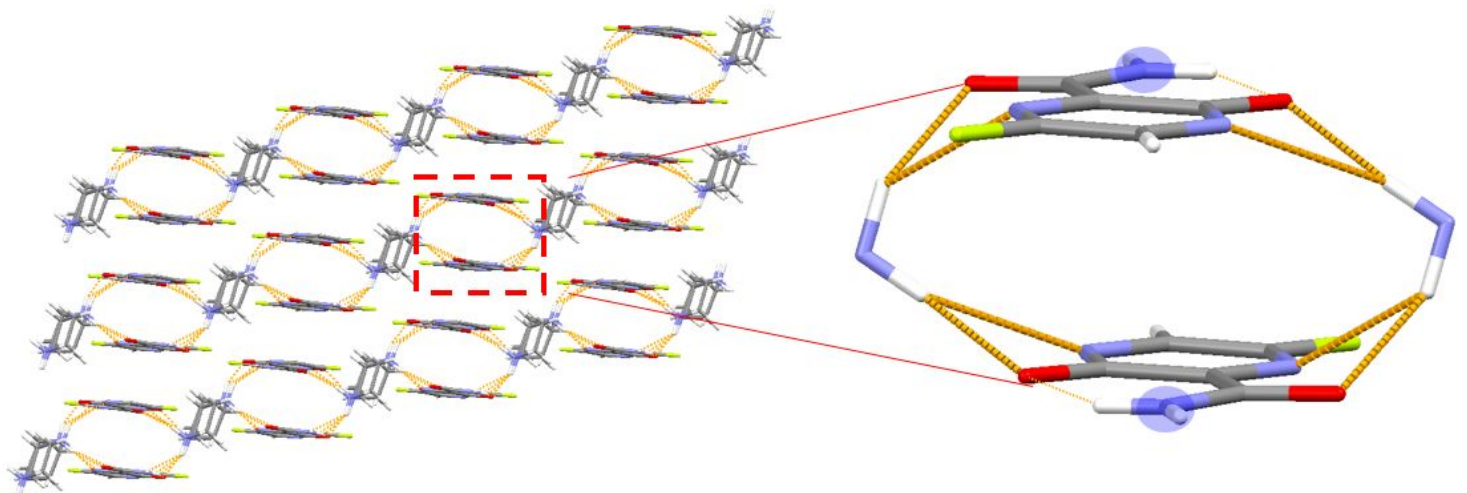

Figure S3. The cavity structure of FPV-PP. The shades of blue correspond to the primary hydrophilic amino groups. 

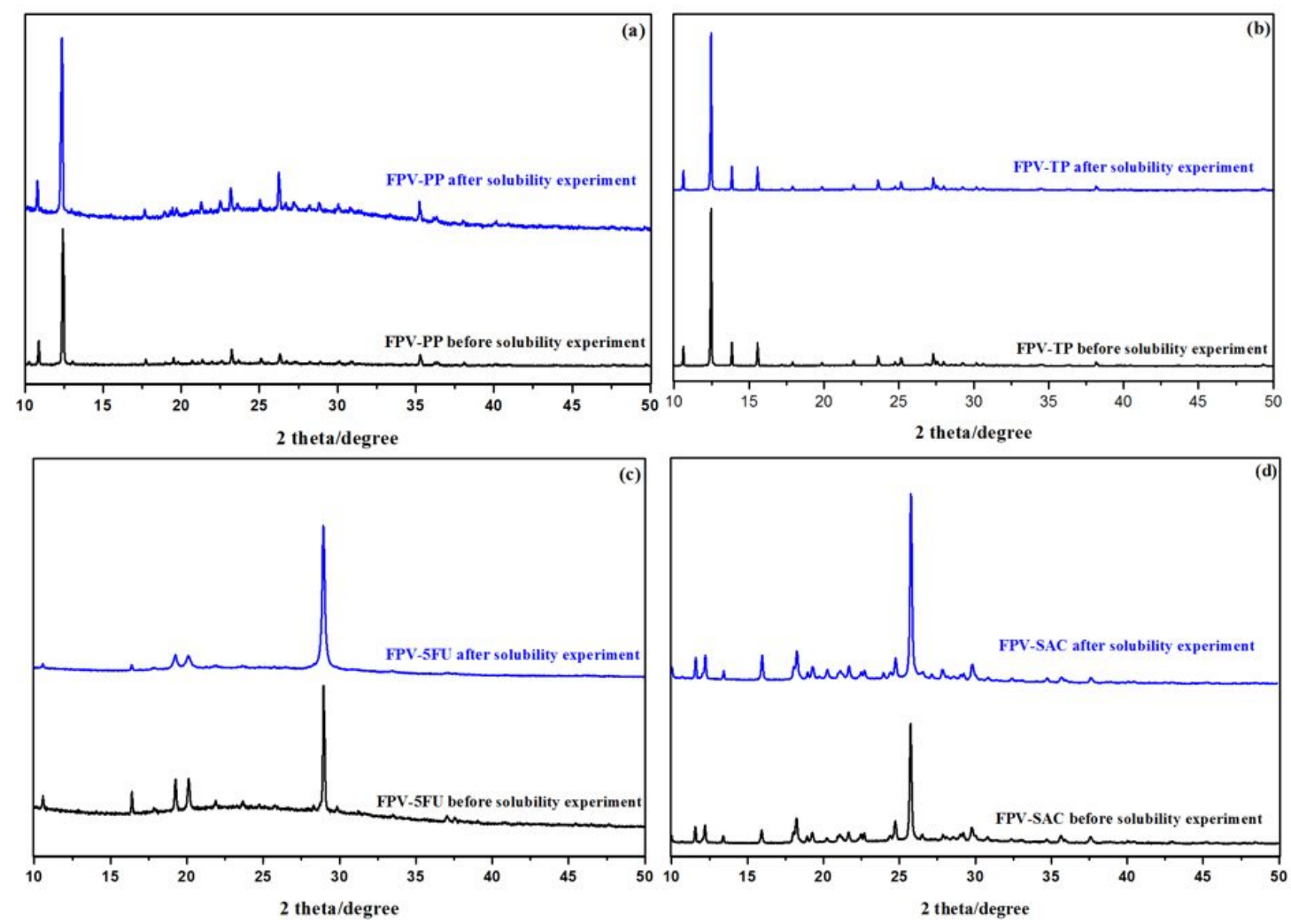

Figure S4. Comparison of XRD patterns of (a) FPV-PP after solubility experimental, FPVPP before solubility experimental; (b) FPV-TP after solubility experimental, FPV-TP before solubility experimental (c) FPV-5FU after solubility experimental, FPV-5FU before solubility experimental (d) FPV-SAC after solubility experimental, FPV-SAC before solubility experimental. 

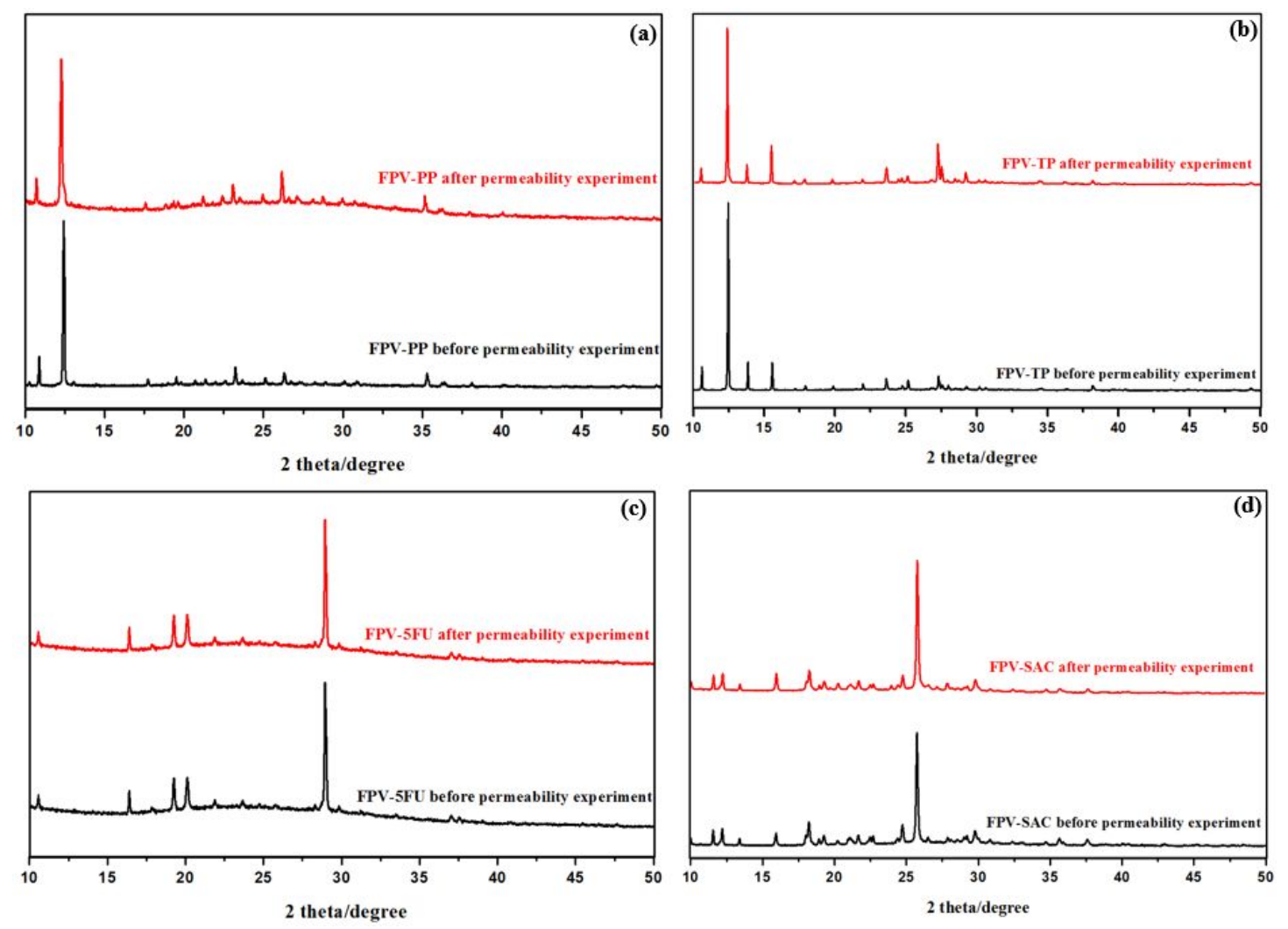

Figure S5. Crystal structure of FPV-PP salt showing slip plane system

Comparison of XRD patterns of (a) FPV-PP after permeability experimental, FPV-PP before permeability experimental; (b) FPV-TP after permeability experimental, FPV-TP before permeability experimental (c) FPV-5FU after permeability experimental, FPV-5FU before permeability experimental (d) FPV-SAC after permeability experimental, FPVSAC before permeability experimental. 


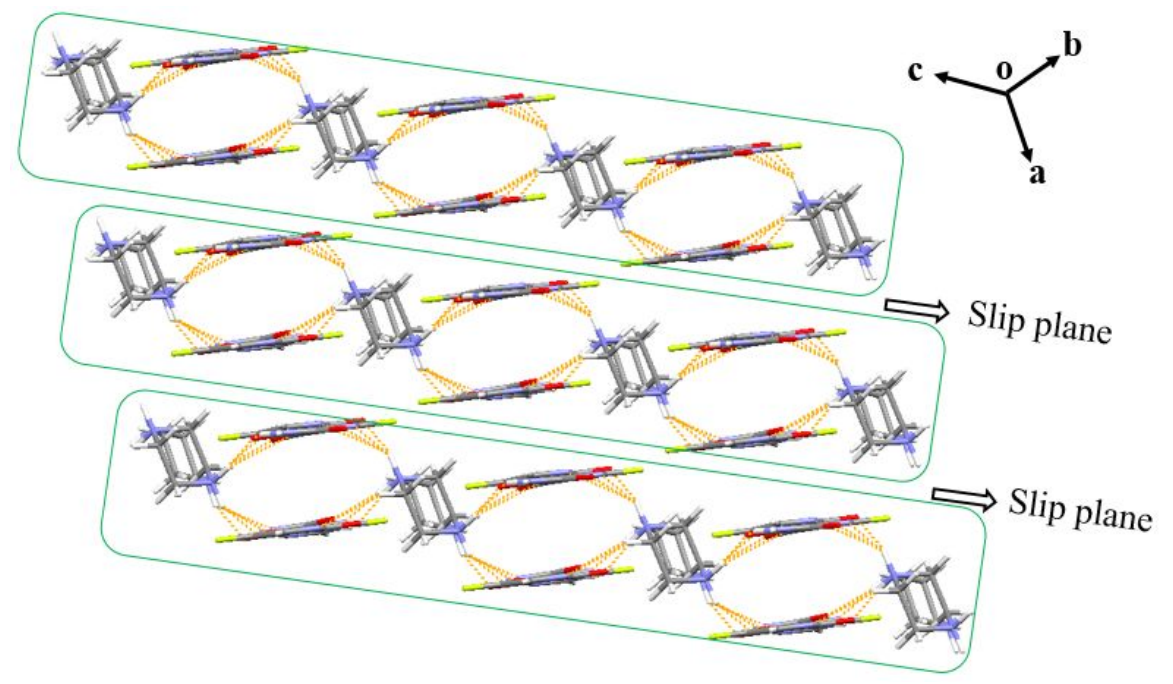

Figure S6. Crystal structure of FPV-PP salt showing slip plane system

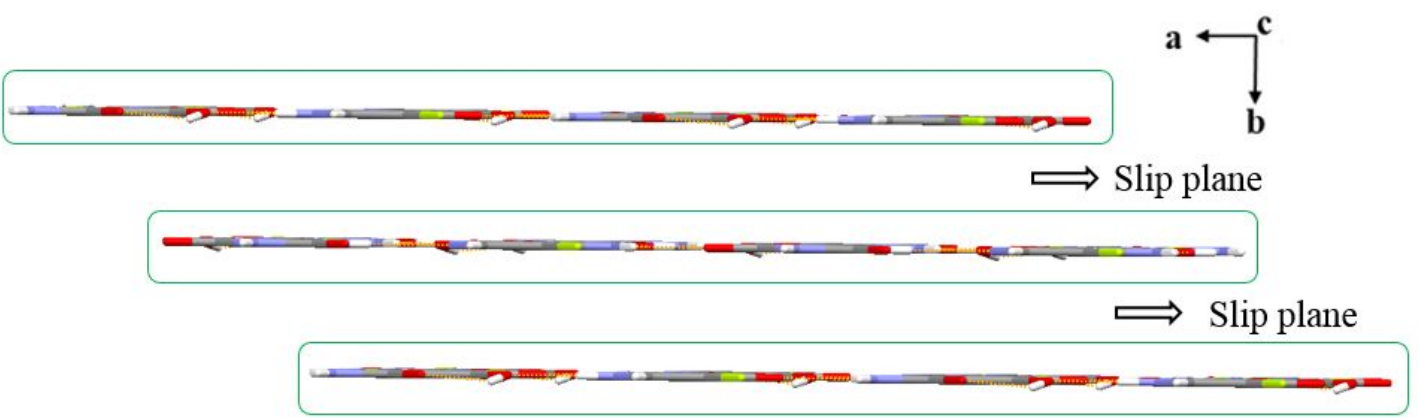

Figure S7. Crystal structure of FPV-5FU cocrystal showing slip plane system. 


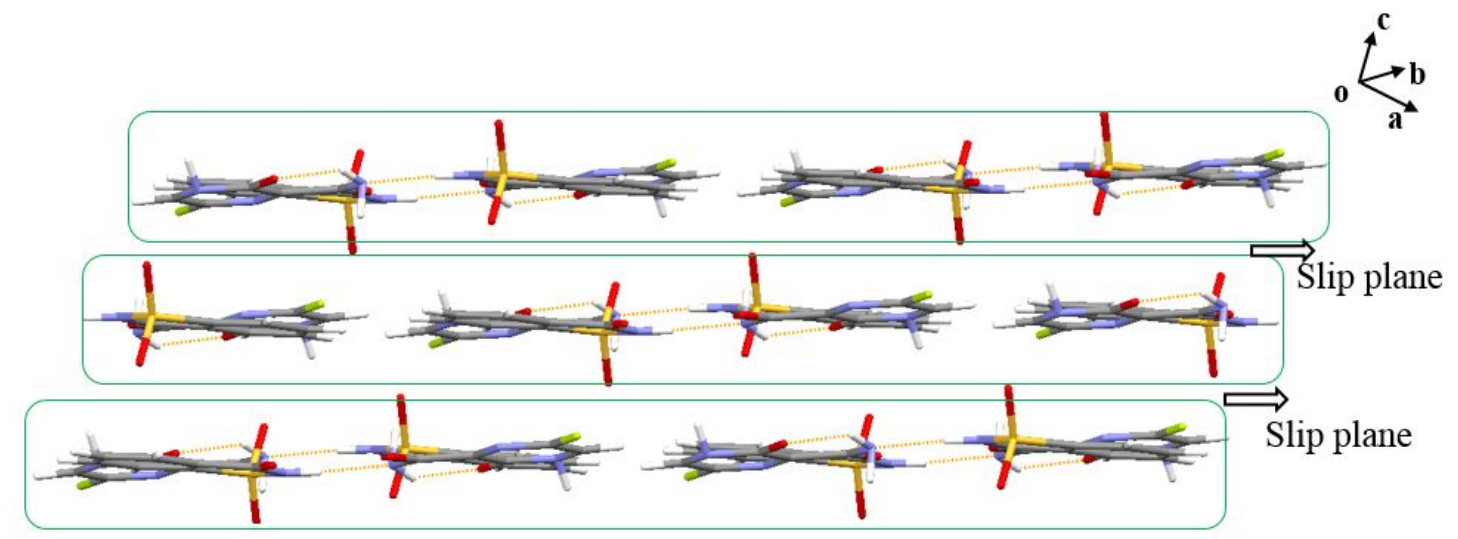

Figure S8. Crystal structure of FPV-SAC cocrystal showing slip plane system.

(a)

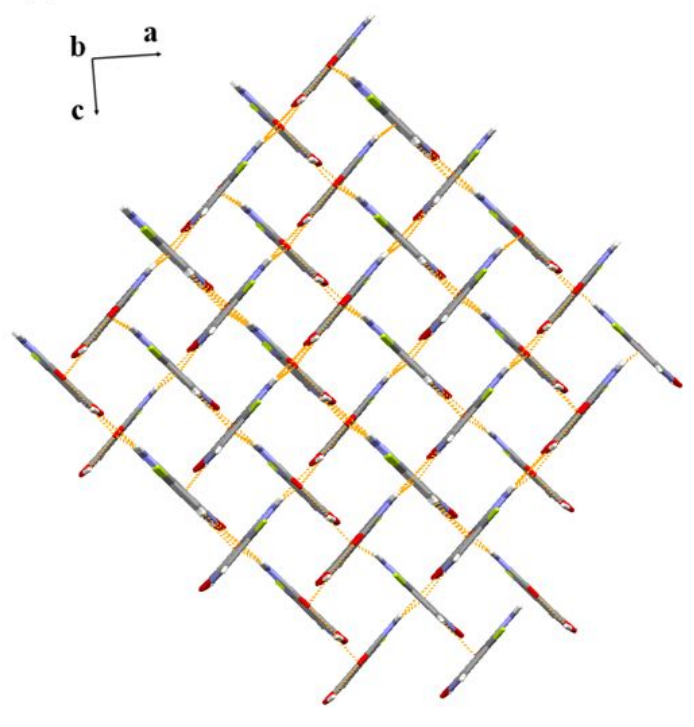

(b)
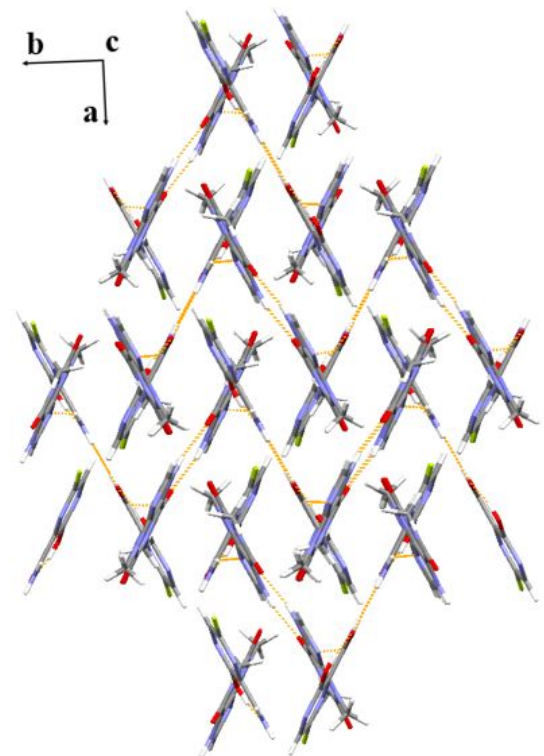

Figure S9. (a) The 3D structure of FPV. (b) The 3D structure of FPV-TP. 


\section{Supplementary Tables}

Table S1 Chromatographic parameters

\begin{tabular}{|l|l|}
\hline Name & Specification \\
\hline Column & $5 \mu \mathrm{m}, 150 \mathrm{~mm} \times 4.6 \mathrm{~mm}$ \\
\hline$\lambda$ max $(\mathrm{nm})$ & 225 \\
\hline Linearity range $(\mathrm{mg} / \mathrm{mL})$ & $0.05-1.5$ \\
\hline Injection volume $(\mu \mathrm{L})$ & 20 \\
\hline Flow $(\mathrm{mL} / \mathrm{min})$ & 1.0 \\
\hline Elution & Isocratic \\
\hline Mobile phase & $\begin{array}{l}0.01 \mathrm{~mol} \cdot \mathrm{L}^{-1} \text { ammonium dihydrogen phosphate } \\
\text { solution }(\text { adjusting } \mathrm{pH} \text { to } 3.2 \text { with trifluoroacetic } \\
\text { acid }): \text { methanol -acetonitrile }(50: 50)=80: 20\end{array}$ \\
\hline
\end{tabular}

Table S2. Thermal Data of FPV, Salt and Co-crystals

\begin{tabular}{|l|l|c|l|c|c|}
\hline \multicolumn{1}{|c|}{ phase } & FPV & FPV-PP & FPV-TP & FPV-5FU & FPV-SAC \\
\hline$T_{\mathrm{m}}\left({ }^{\circ} \mathrm{C}\right)$ & 188.32 & - & 193.64 & - & - \\
\hline$\Delta H \mathrm{~m}(\mathrm{~J} / \mathrm{g})$ & -147.21 & - & -104.44 & - & - \\
\hline $\mathrm{T}_{\mathrm{dec}}\left({ }^{\circ} \mathrm{C}\right)$ & 189.07 & 215.76 & 194.16 & 210.79 & 150.93 \\
\hline
\end{tabular}


Table S3. The $\mathrm{pH}$ variation of the solution after solubility and permeability experiments $(n=3)$

\begin{tabular}{|c|c|c|}
\hline Samples & $\begin{array}{l}\text { pH values of the buffer (after } \\
\text { the solubility experimental) }\end{array}$ & $\begin{array}{l}\mathrm{pH} \text { values of the buffer (after the } \\
\text { permeability xperimental) }\end{array}$ \\
\hline FPV & $5.545 \pm 0.02$ & $6.207 \pm 0.02$ \\
\hline FPV-PP & $6.31 \pm 0.03$ & $6.421 \pm 0.02$ \\
\hline FPV-TP & $5.62 \pm 0.02$ & $6.450 \pm 0.03$ \\
\hline FPV-5FU & $5.89 \pm 0.02$ & $6.105 \pm 0.01$ \\
\hline FPV-SAC & $5.79 \pm 0.02$ & $5.812 \pm 0.01$ \\
\hline
\end{tabular}

Table S4 Experimental conditions for studying compressibility ( $\mathrm{n}=3$ )

\begin{tabular}{|c|c|c|c|}
\hline Sample amount & \multicolumn{3}{|c|}{$120 \mathrm{mg}$} \\
\hline Sample code & A & B & $45 \mathrm{~s}$ \\
\hline Dwell time & $15 \mathrm{~s}$ & $30 \mathrm{~s}$ \\
\hline \multicolumn{2}{|c|}{ Compression using $10 \mathrm{~mm}$ die at $25 \mathrm{~kg} / \mathrm{cm}^{2}$ pressure } \\
\hline \multicolumn{2}{|c|}{ Equilibration in closed glass vials for 24 hour } \\
\hline
\end{tabular}


Table S5 Flow properties and compressibility parameters $(n=3)$

\begin{tabular}{|l|c|c|c|}
\hline Drug & Angle of repose ( $)$ & Carr's index (\%) & Housner's ratio \\
\hline FPV & $53.48 \pm 2.05$ & 28.74 & 1.40 \\
\hline FPV-PP & $55.8 \pm 0.89$ & 24.81 & 1.33 \\
\hline FPV-TP & $41.32 \pm 0.33$ & 18.70 & 1.23 \\
\hline FPV-5FU & $48.87 \pm 1.86$ & 12.28 & 1.14 \\
\hline FPV-SAC & $45.05 \pm 0.5$ & 10.71 & 1.24 \\
\hline
\end{tabular}

